\title{
Entanglement entropy and its quench dynamics for pure states of the Sachdev-Ye-Kitaev model
}

\author{
Pengfei Zhang \\ Walter Burke Institute for Theoretical Physics, California Institute of Technology, \\ Pasadena, CA 91125, U.S.A. \\ Institute for Quantum Information and Matter, California Institute of Technology, \\ Pasadena, CA 91125, U.S.A. \\ E-mail: pengfeizhang.physics@gmail.com
}

\begin{abstract}
Sachdev-Ye-Kitaev (SYK) is a concrete solvable model with non-Fermi liquid behavior and maximal chaos. In this work, we study the entanglement Rényi entropy for the subsystems of the SYK model in the Kourkoulou-Maldacena states. We use the path-integral approach and take the saddle point approximation in the large- $N$ limit. We find a first-order transition exist when tuning the subsystem size for the $q=4$ case, while it is absent for the $q=2$ case. We further study the entanglement dynamics for such states under the real-time evolution for noninteracting, weakly interacting and strongly interacting SYK(-like) models.
\end{abstract}

KeYwords: 1/N Expansion, Field Theories in Lower Dimensions, Models of Quantum Gravity

ArXiv ePrint: 2004.05339 


\section{Contents}

1 Introduction 1

2 SYK model and Kourkoulou-Maldacena states 2

3 Path-integral for pure-state entanglement entropy $\quad 4$

3.1 A warm up: $\langle\mathrm{KM} \mid \mathrm{KM}\rangle \quad 4$

$\begin{array}{ll}3.2 \text { Computing } \mathcal{S}_{A}^{(n)} & 7\end{array}$

$\begin{array}{llr}4 & \text { Numerical results } & 8\end{array}$

5 Quench dynamics for pure-state entanglement entropy 10

$\begin{array}{llr}6 \text { Conclusion } & 15\end{array}$

\section{Introduction}

In recent years, the entanglement entropy and its dynamics in many-body systems have drawn a lot of attention. As an example, the entanglement entropy has been studied both theoretically [1-4] and experimentally $[5,6]$ for interacting quantum systems that satisfy the eigenstate thermalization hypothesis (ETH). For a general energy eigenstate, it shows volume law scaling, in contrast to the area law scaling in the many-body localization (MBL) phase $[7,8]$. The entanglement dynamics can also be related to the out-of-time-order correlators $[9,10]$, which characterize the scrambling of quantum information [11-14]. Moreover, the recent resolution of the information paradox [15-20] is directly from the refined understanding of the Ryu-Takayanagi formula [21-23] for computing the entanglement entropy in holographic systems.

Unfortunately, the calculation of the entanglement entropy for many-body systems is usually hard. Toy models where entanglement entropy can be computed efficiently are of especial interest. One strategy is to construct random unitary dynamics [24-26]. Here, we consider an alternative route by studying a specific solvable model named the Sachdev-YeKitaev model [12, 27-29], which describes $N$ Majorana modes with infinite range $q$-body interaction. For $q \geq 4$, it is known as a non-Fermi liquid without quasiparticles which shows maximal chaotic behavior. In the low-energy limit, the system is described in terms of reparametrization modes, with an effective Schwarzian action [12, 28, 29]. The same action also shows up for the Jackiw-Teitelboim gravity in 2D [29, 30].

Previously, there are studies of the SYK model from the entropy perspective. Assuming the system satisfies ETH [1, 2, 31-33], the entanglement entropy can be related to the thermal entropy with an effective temperature depending on the system size [34]. An analytical approximation has also been derived based on the many-body spectrum [35]. 
Numerically, the subsystem entropy has been studied in [36] by exact diagonalization for the ground state, and in [37, 38] by path-integral approach for thermal ensembles. There are also studies for two copies of (coupled) SYK models prepared in the thermofield double state, which purifies the thermal density matrix [20,39,40]. There are also studies for random hopping model, which is directly related to the $\mathrm{SYK}_{2}$ case [41, 42]. However, the large- $N$ microscopic entanglement entropy and its dynamics for pure states of a single SYK model are still unknown. ${ }^{1}$

In this work, we establish the formulation for computing Rényi entanglement entropy for the Kourkoulou-Maldacena pure states in SYK-like models [44]. The paper is organized as follows: in section 2, we give a brief review of the SYK model and the KourkoulouMaldacena states. We then derive the path-integral representation of Rényi entanglement entropy for such pure states in section 3. The numerical results are presented in section 4 . By varying the subsystem size, we find a first-order transition of the entanglement entropy for the $\mathrm{SYK}_{4}$ model, which leads to the singularity of the Page curve at half system size [45]. This is qualitatively different from the $q=2$ case, where the entanglement entropy changes analytically when varying the subsystem size. We further study the exact dynamics of the entanglement entropy for the $\mathrm{SYK}_{4}$ non-Fermi liquid, with a comparison to noninteracting or weakly interacting $\mathrm{SYK}_{2}$ Fermi liquids in section 5. Finally, we summarize our results in 6 .

\section{SYK model and Kourkoulou-Maldacena states}

The Hamiltonian of $\mathrm{SYK}_{q}$ model $[12,28]$ reads:

$$
H=\frac{1}{q !} \sum_{i_{1} i_{2} \ldots i_{q}} i^{q / 2} J_{i_{1} i_{2} \ldots i_{q}}^{(q)} \chi_{i_{1}} \chi_{i_{2}} \ldots \chi_{i_{q}} .
$$

Here $q$ is an even integer and $i=1,2 \ldots N$ labels different Majorana modes. We take the convention that $\left\{\chi_{i}, \chi_{j}\right\}=\delta_{i j} . J_{i_{1} i_{2} \ldots i_{q}}^{(q)}$ are independent Gaussian variables with:

$$
\overline{\left|J_{i_{1} i_{2} \ldots i_{q}}^{(q)}\right|}=0, \quad \overline{\left|J_{i_{1} i_{2} \ldots i_{q}}^{(q)}\right|^{2}}=\frac{(q-1) ! J^{2}}{N^{q-1}}=\frac{2^{q-1}(q-1) ! \mathcal{J}^{2}}{q N^{q-1}} .
$$

Here $\mathcal{J}$ is taken to be a constant in the large- $q$ limit.

For a thermal ensemble, to the leading order of $1 / N$, the two-point correlator $G_{\mathrm{th}}(\tau)=$ $\left\langle\mathcal{T}_{\tau} \chi_{i}(\tau) \chi_{i}(0)\right\rangle_{\beta}$ satisfies the self-consistent equation:

$$
G_{\mathrm{th}}^{-1}\left(i \omega_{n}\right)=-i \omega_{n}-\Sigma_{\mathrm{th}}\left(i \omega_{n}\right), \quad \Sigma_{\mathrm{th}}(\tau)=\stackrel{\cdots}{\vdots}=J^{2} G_{\mathrm{th}}^{q-1}(\tau)
$$

where the self-energy is given by melon diagrams. By solving the Schwinger-Dyson equation, the model is found to be a Fermi liquid with finite spectral function near $\omega \sim 0$ for $q=2$. On contrary, for $q \geq 4$, the model has divergent spectral function $\rho(\omega) \sim \omega^{2 / q-1}$,

\footnotetext{
${ }^{1}$ There are related dicussions of entanglement entropy in [43].
} 
which is known as a non-Fermi liquid. Further study shows it has maximal chaos $[12,28]$ and satisfies the ETH [31-33].

Considering the SYK system in some eigenstate $|E\rangle$ with energy $E=N \epsilon$, the entropy $\mathcal{S}_{A}$ of the a subsystem $A$ containing $M=\lambda N(\lambda<1 / 2)$ Majorana fermions is argued to be [34]:

$$
\mathcal{S}_{A}=M s_{\mathrm{th}}\left(\lambda^{\frac{q-1}{2}} \epsilon\right),
$$

for $q \geq 4$. Here $s_{\mathrm{th}}(x)$ is the thermal entropy density in the micro-canonical ensemble with energy density $x$. A similar statement when the total system is prepared in a thermal ensemble has been tested in [37]. Approximately, we have $s_{\text {th }}(x) \approx$ $\left(\log (2) / 2-\arcsin \left(x / \epsilon_{0}\right) / q^{2}\right)$ with $\epsilon_{0}$ being the energy density of the ground state [35]. On the other hand, for $q=2$, the ground state entanglement entropy can be calculated analytically [36, 37].

In this work, we focus on a specific class of pure states of the SYK model [44]. These states are now known as the Kourkoulou-Maldacena (KM) states. To construct them, we first pair Majorana fermions as $c_{j}=\frac{\chi_{2 j-1}+i \chi_{2 j}}{2}$ with $j=1,2 \ldots N / 2 .^{2}$ Then (unnormalized) KM states are given by:

$$
|\operatorname{KM}(\{s\}, \beta)\rangle=e^{-\frac{\beta H}{2}}|\{s\}\rangle, \quad\left(2 n_{j}-1\right)|\{s\}\rangle=s_{j}|\{s\}\rangle .
$$

Here $n_{j}=c_{j}^{\dagger} c_{j}$ and $s_{j} \in\{ \pm 1\}$. We could always redefine $\chi_{2 j} \rightarrow s_{j} \chi_{2 j}$ to set $s_{j}=1$ for all $j$. As result, all single states are equivalent after averaging over the ensemble of random interaction. We will focus on $\{s\}=\{1\}$ for most parts of the manuscript. Moreover, for simplicity, from now on we keep the $\beta=1 / T$ dependence of $|\mathrm{KM}\rangle$ implicit.

The hallmark of these states is that to the leading order of $1 / N$, the correlation functions of $\chi_{i}$ can be related to thermal correlators [44], under the assumption of the disorder replica diagonal [29, 46-48]. As an example, two-point functions

$$
G_{i j}\left(\tau, \tau^{\prime}\right)=\frac{1}{Z_{\mathrm{KM}}}\left\langle\{1\}\left|\mathcal{T}_{\tau} e^{-\int_{0}^{\beta} d \tau H} \chi_{i}(\tau) \chi_{i}\left(\tau^{\prime}\right)\right|\{1\}\right\rangle,
$$

with $Z_{\mathrm{KM}} \equiv\langle\mathrm{KM} \mid \mathrm{KM}\rangle \equiv e^{-I_{\mathrm{KM}}}$, can be expressed in terms of the thermal Green's function $G_{\mathrm{th}}(\tau)$. Explicitly, all non-zero components are

$$
G_{i i}\left(\tau, \tau^{\prime}\right)=G_{\mathrm{th}}\left(\tau-\tau^{\prime}\right), \quad G_{2 j-1,2 j}\left(\tau, \tau^{\prime}\right)=-i 2 G_{\mathrm{th}}(\tau) G_{\mathrm{th}}\left(\tau^{\prime}\right) .
$$

Here we have $\tau, \tau^{\prime} \in[0, \beta]$. This shows that the diagonal component $G_{i i}$ take the same form as a thermal Green's function, while the off-diagonal part $G_{2 j-1,2 j}(\beta / 2, \beta / 2)$ characterize the deviation from a thermalized state (at the two-point function level). For $\beta J \rightarrow \infty,|\mathrm{KM}\rangle$ selects one state from the ground state sector of the SYK model, and $G_{2 j-1,2 j}(\beta / 2, \beta / 2) \rightarrow 0$.

We are mainly interested in the Rényi entanglement entropy of such pure states. For such states, we define subsystem A consisting of $M / 2$ complex fermions. ${ }^{3}$ The reduced density matrix $\rho_{A}=\frac{1}{Z_{\mathrm{KM}}} \operatorname{tr}_{B}|\mathrm{KM}\rangle\langle\mathrm{KM}| \equiv \tilde{\rho}_{A} / Z_{\mathrm{KM}}$ is given by tracing out its complimentary

\footnotetext{
${ }^{2}$ Since there is a permutation symmetry for different modes $i$, this choice is general.

${ }^{3}$ Note that although the KM pure states are expected to be dual to an $\mathrm{AdS}_{2}$ geometry with a brane, there is no index $i$ degree of freedom, and consequently no direct analogy of this entanglement entropy in the gravity picture.
} 
$B$. The $n$-th Rényi entropy is then given by

$$
\mathcal{S}_{A}^{(n)}=\frac{1}{1-n} \log \left(\operatorname{tr}_{A} \rho_{A}^{n}\right),
$$

with $\mathcal{S}_{A} \equiv \mathcal{S}_{A}^{(1)}$ being the Von Neumann entropy. Since the full system is in a pure state, we expect $\mathcal{S}_{A}^{(n)}$ being symmetric under a reflection along $\lambda=1 / 2$. For $\beta=0,|\mathrm{KM}\rangle$ is a product state, and we have $\mathcal{S}_{A}^{(n)}=0$. On the contrary, if we take $\beta J \rightarrow \infty$, and we expect $\mathcal{S}_{A}^{(n)}$ follow a Page curve [45] with energy density depending on subsystem size (2.4).

\section{Path-integral for pure-state entanglement entropy}

In this section, we derive the path-integral representation of $\mathcal{S}_{A}^{(n)}$ for KM pure states. We begin with a warm up by computing the normalization factor $Z_{\mathrm{KM}}$ in section 3.1, which is also needed then computing the entanglement entropy. The path integral formula for computing $\mathcal{S}_{A}^{(n)}$ is then derived in 3.2.

\subsection{A warm up: $\langle\mathrm{KM} \mid \mathrm{KM}\rangle$}

Let us first consider the path-integral representation of $Z_{\mathrm{KM}}=e^{-I_{\mathrm{KM}}}=\langle\mathrm{KM} \mid \mathrm{KM}\rangle$. Note that this is in fact not essential, since $Z_{\mathrm{KM}}$ can be directly related to the thermal partition function $Z=\operatorname{tr} e^{-\beta H}$ [37]. However, the trick developed in this subsection is useful when computing the entanglement entropy.

The state $|\mathrm{KM}\rangle$ is given by an imaginary-time evolution of a initial state $|\{1\}\rangle$. The graphic representation is:

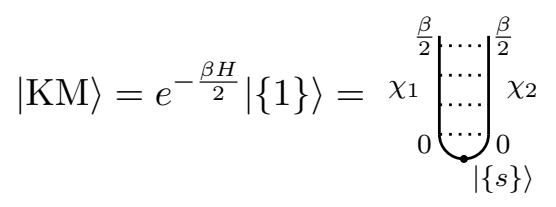

Here we have explicitly separated out fermions with odd/even indices: $\chi_{1 / 2}$ represents Majorana fermions $\chi_{2 j-1} / \chi_{2 j}$ with odd/even indices. The solid lines denote the imaginarytime evolution and the dotted lines represent interactions between fermions. Two points connected by the dotted line are at the same imaginary time. The black dots represent the boundary condition $c_{j}|\{1\}\rangle=0$, or in terms of Majorana fermions $\left(\chi_{2 j-1}+i \chi_{2 j}\right)|\{1\}\rangle=0$. Similarly, the normalization $Z_{\mathrm{KM}}$ is given by

$$
Z_{\mathrm{KM}}=e^{-I_{\mathrm{KM}}}=\left\langle\{1\}\left|e^{-\beta H}\right|\{1\}\right\rangle=\left.\chi_{1} \underbrace{\beta}_{0} \ldots\right|^{\langle\{s\}|} \chi_{02}^{\beta}
$$

Here we have another boundary condition $\langle\{1\}|\left(\chi_{2 j-1}-i \chi_{2 j}\right)=0$ at imaginary time $\beta$. The path-integral representation of $Z_{\mathrm{KM}}$ is then

$$
\begin{aligned}
e^{-I_{\mathrm{KM}}} & =\int_{\text {b.c. }} \mathcal{D} \chi_{i}(\tau) \exp \left(-S_{\mathrm{KM}}\left[\chi_{i}\right]\right), \\
S_{\mathrm{KM}} & =\int_{0}^{\beta} d \tau\left(\frac{1}{2} \sum_{i} \chi_{i} \partial_{\tau} \chi_{i}+\frac{1}{q !} \sum_{i_{1} i_{2} \ldots i_{q}} i^{q / 2} J_{i_{1} i_{2} \ldots i_{q}}^{(q)} \chi_{i_{1}} \chi_{i_{2}} \ldots \chi_{i_{q}}\right) .
\end{aligned}
$$


Here b.c. indicates the boundary condition at $\tau=0$ and $\tau=\beta$ :

$$
\chi_{2 j-1}(0)=-i \chi_{2 j}(0), \quad \chi_{2 j-1}(\beta)=i \chi_{2 j}(\beta) .
$$

We further take the disorder average of random interaction $J_{i_{1} i_{2} \ldots i_{q}}^{(q)}$. As for the thermal ensemble, we expect the replica diagonal assumption works well in the large- $N$ limit and we could neglect the difference between $\exp \left(-\overline{I_{\mathrm{KM}}}\right)$ and $\overline{\exp \left(-I_{\mathrm{KM}}\right)}$. Consequently, we keep the disorder average implicitly from now on.

To proceed, we use the standard trick by introducing bilocal fields $G$ and $\Sigma$ [28]. Since fields with even or odd indices are in-equivalent, we should define two sets of fields $G_{11 / 22}$ and $\Sigma_{11 / 22}$. The definition of $G_{11}$ and $G_{22}$ is

$$
G_{11}\left(\tau, \tau^{\prime}\right)=\frac{2}{N} \sum_{j} \chi_{2 j-1}(\tau) \chi_{2 j-1}\left(\tau^{\prime}\right), \quad G_{22}\left(\tau, \tau^{\prime}\right)=\frac{2}{N} \sum_{j} \chi_{2 j}(\tau) \chi_{2 j}\left(\tau^{\prime}\right) .
$$

$\Sigma_{11}$ and $\Sigma_{22}$ are introduced in order to impose the relation between $G$ and $\chi \chi$ :

$$
\begin{aligned}
\delta\left(G_{11}-\frac{2}{N} \sum_{i \in \text { odd }} \chi_{i} \chi_{i}\right) & =\int \mathcal{D} \Sigma_{22} e^{\frac{1}{2} \int d \tau d \tau^{\prime} \Sigma_{11}\left(\tau, \tau^{\prime}\right)\left(\sum_{\text {odd }} \chi_{i}(\tau) \chi_{i}\left(\tau^{\prime}\right)-\frac{N}{2} G_{11}\left(\tau, \tau^{\prime}\right)\right)}, \\
\delta\left(G_{22}-\frac{2}{N} \sum_{i \in \text { even }} \chi_{i} \chi_{i}\right) & =\int \mathcal{D} \Sigma_{22} e^{\frac{1}{2} \int d \tau d \tau^{\prime} \Sigma_{22}\left(\tau, \tau^{\prime}\right)\left(\sum_{\text {even }} \chi_{i}(\tau) \chi_{i}\left(\tau^{\prime}\right)-\frac{N}{2} G_{22}\left(\tau, \tau^{\prime}\right)\right) .}
\end{aligned}
$$

Then by integrating out the Majorana fields, we find

$$
e^{-I_{\mathrm{KM}}}=\int \mathcal{D} G_{11} \mathcal{D} G_{22} \mathcal{D} \Sigma_{11} \mathcal{D} \Sigma_{22} \exp \left(-S_{\mathrm{KM}}^{\mathrm{eff}}[G, \Sigma]\right) .
$$

Here the effective $G-\Sigma$ action $S_{\mathrm{KM}}$ is given by ${ }^{4}$

$$
\begin{aligned}
\frac{S_{\mathrm{KM}}^{\mathrm{eff}}}{N}= & -\frac{1}{4} \log \underset{\text { b.c. }}{\operatorname{det}}\left(\begin{array}{cc}
\partial_{\tau}-\Sigma_{11} & 0 \\
0 & \partial_{\tau}-\Sigma_{22}
\end{array}\right)-\frac{J^{2}}{2 q} \int d \tau d \tau^{\prime}\left(\frac{G_{11}\left(\tau, \tau^{\prime}\right)+G_{22}\left(\tau, \tau^{\prime}\right)}{2}\right)^{q} \\
& +\frac{1}{4} \int d \tau d \tau^{\prime} G_{11}\left(\tau, \tau^{\prime}\right) \Sigma_{11}\left(\tau, \tau^{\prime}\right)+\frac{1}{4} \int d \tau d \tau^{\prime} G_{22}\left(\tau, \tau^{\prime}\right) \Sigma_{22}\left(\tau, \tau^{\prime}\right) .
\end{aligned}
$$

Here b.c. denotes the boundary condition 3.4. Note that although the self-energy is blocked diagonal, the boundary condition would mix modes with even/odd indices. In the large- $N$ limit, we take the saddle point of (3.8). The saddle point equation reads

$$
\begin{gathered}
\left(\begin{array}{ll}
G_{11} & G_{12} \\
G_{21} & G_{22}
\end{array}\right)=\left(\begin{array}{cc}
\partial_{\tau}-\Sigma_{11} & 0 \\
0 & \partial_{\tau}-\Sigma_{22}
\end{array}\right)_{\text {b.c. }}^{-1}, \\
\Sigma_{11}\left(\tau, \tau^{\prime}\right)=\Sigma_{22}\left(\tau, \tau^{\prime}\right)=J^{2}\left(\frac{G_{11}\left(\tau, \tau^{\prime}\right)+G_{22}\left(\tau, \tau^{\prime}\right)}{2}\right)^{q-1} .
\end{gathered}
$$

\footnotetext{
${ }^{4}$ There could be additional boundary terms. Nevertheless, they cancel out when computing the entanglement entropy.
} 
Here we have also introduced

$$
G_{12}\left(\tau, \tau^{\prime}\right)=\frac{2}{N} \sum_{j}\left\langle\mathcal{T}_{\tau} \chi_{2 j-1}(\tau) \chi_{2 j}\left(\tau^{\prime}\right)\right\rangle, \quad G_{21}\left(\tau, \tau^{\prime}\right)=\frac{2}{N} \sum_{j}\left\langle\mathcal{T}_{\tau} \chi_{2 j}(\tau) \chi_{2 j-1}\left(\tau^{\prime}\right)\right\rangle,
$$

for completeness. In terms of bilocal fields $G_{a b}$ with $a, b \in\{1,2\}$, the boundary condition (3.4) becomes

$$
\begin{array}{llrl}
G_{1 a}(0, \tau) & =-i G_{2 a}(0, \tau), & & G_{1 a}(\beta, \tau)=i G_{2 a}(\beta, \tau), \\
G_{a 1}(\tau, 0) & =-i G_{a 2}(\tau, 0), & & G_{a 1}(\tau, \beta)=i G_{a 2}(\tau, \beta) .
\end{array}
$$

Solving the equation (3.9) with boundary condition (3.10), and substituting the solution into (3.8) already gives the on-shell action $I_{\mathrm{KM}}$ and thus $Z_{\mathrm{KM}}$. However, it is more convenient to introduce a different parametrization of the contour. The key observation is that if we define a Majorana field $\chi_{j}(s)$ with parameter $s \in[0,2 \beta)$ :

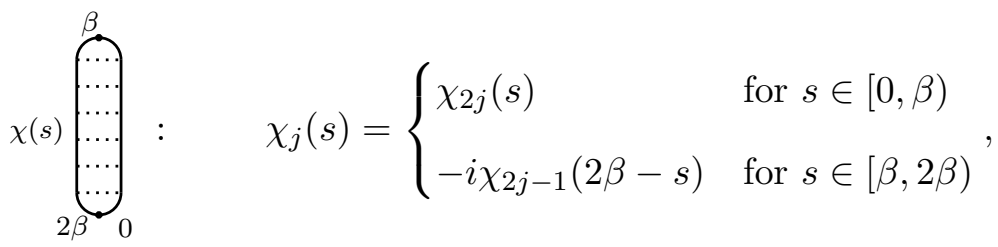

here $j=1,2 \ldots N / 2$, the boundary condition (3.4) becomes the traditional continuous and anti-periodic boundary condition $\chi_{j}\left(2 \beta^{-}\right)=-\chi_{j}\left(0^{+}\right)$, as for a thermal ensemble. The Green's function $G\left(s, s^{\prime}\right)=\left\langle\mathcal{T}_{\mathcal{C}} \chi_{j}(s) \chi_{j}\left(s^{\prime}\right)\right\rangle$ is then given by

$$
G\left(s, s^{\prime}\right)=\left(\begin{array}{cc}
G_{22}\left(s, s^{\prime}\right) & -i G_{21}\left(s, 2 \beta-s^{\prime}\right) \\
-i G_{12}\left(2 \beta-s, s^{\prime}\right) & -G_{11}\left(2 \beta-s, 2 \beta-s^{\prime}\right)
\end{array}\right) .
$$

The self-consistent equation for $G\left(s, s^{\prime}\right)$ is then

$$
G\left(s, s^{\prime}\right)=\left(\partial_{s}-\Sigma\right)^{-1}\left(s, s^{\prime}\right) \equiv\left(\begin{array}{cc}
\partial_{s}-\Sigma_{22}\left(s, s^{\prime}\right) & 0 \\
0 & \partial_{s}+\Sigma_{11}\left(2 \beta-s, 2 \beta-s^{\prime}\right)
\end{array}\right)^{-1} .
$$

Moreover, both the action (3.8) and boundary condition (3.4) are invariant under $\chi_{2 j-1} \rightarrow$ $\chi_{2 j}$ and $\chi_{2 j} \rightarrow-\chi_{2 j-1}$. As a result, we have $G_{11}\left(\tau, \tau^{\prime}\right)=G_{22}\left(\tau, \tau^{\prime}\right)$. Instead of (3.9), we can then use

$$
\begin{aligned}
& \Sigma\left(s, s^{\prime}\right)=J^{2} G^{q-1}\left(s, s^{\prime}\right) P\left(s, s^{\prime}\right), \\
& P\left(s, s^{\prime}\right)=\left[\theta(\beta-s) \theta\left(\beta-s^{\prime}\right)+\theta(s-\beta) \theta\left(s^{\prime}-\beta\right)\right] .
\end{aligned}
$$

Here $P\left(s, s^{\prime}\right)$ is a projector. We have $P\left(s, s^{\prime}\right)=1$ if both $\chi(s)$ and $\chi\left(s^{\prime}\right)$ represents the same field $\left(\chi_{1}\right.$ or $\left.\chi_{2}\right)$ and otherwise zero. This definition for $P\left(s, s^{\prime}\right)$ is more general if we choose a different $s=0$ point on the contour (3.11). Note that comparing to a thermofield double state with inverse temperature $2 \beta$ and $N / 2$ fermions, the main difference is the presence of $P\left(s, s^{\prime}\right)$, which breaks the time translational invariance.

We can then choose to solve the equation (3.14) and $G=\left(\partial_{s}-\Sigma\right)^{-1}$ self-consistently. Moreover, we could also express the on-shell action in terms of $G\left(s, s^{\prime}\right)$ and $\Sigma\left(s, s^{\prime}\right)$ :

$$
\frac{I_{\mathrm{KM}}}{N}=\frac{1}{4} \log \operatorname{det} G+\frac{q-1}{4 q} \int d s d s^{\prime} G\left(s, s^{\prime}\right) \Sigma\left(s, s^{\prime}\right) .
$$

This gives an alternative route to compute $Z_{\mathrm{KM}}$. 


\subsection{Computing $\mathcal{S}_{A}^{(n)}$}

Having illustrated the trick of parameterizing the contour by $s$, we consider the pathintegral representation of $\mathcal{S}_{A}^{(n)}$ in this subsection.

To compute $\mathcal{S}_{A}^{(2)}$, we first consider the path-integral representation of $|\mathrm{KM}\rangle\langle\mathrm{KM}|$. Separating out the modes in system $A$ and $B$, a graphic representation is

$$
\left.|\mathrm{KM}\rangle\langle\mathrm{KM}|=| \mathrm{KM}\rangle\right|_{1}
$$

here the red/blue solid line represents the contour for subsystem $A / B . \quad \chi_{1 / 2}^{S}$ represents Majorana fermions in subsystem $S$ with odd/even indices, with $S \in\{A, B\}$.

The unnormalized density matrix $\tilde{\rho}_{A}=\operatorname{tr}_{B}|\mathrm{KM}\rangle\langle\mathrm{KM}|$ is then given by tracing out the $B$ subsystem or, graphically, by connecting the (blue) contours of $B \cdot \operatorname{tr}_{A} \tilde{\rho}_{A}^{n}$ can then be computed by sewing $n$ copies of $\tilde{\rho}_{A}$. To be concrete, in this work we focus on the $n=2$ case. The corresponding contour is then given by

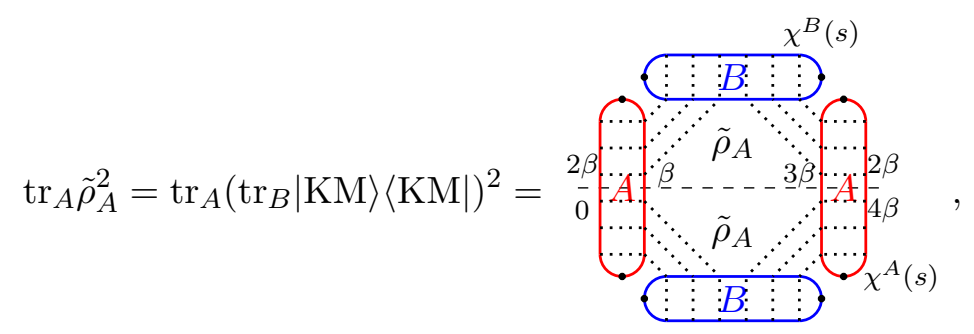

where the symmetry of interchanging $A$ and $B$ becomes obvious. Here we have parametrized the contour by $s \in[0,4 \beta]$ anticlockwise. We define $\chi^{S}(s) \propto \chi_{2}^{S}(s)$ for $s \in[\beta / 2,3 \beta / 2] \cup[5 \beta / 2,7 \beta / 2]$ and $\chi^{S}(s) \propto \chi_{1}^{S}(s)$ otherwise. Similar to the previous section, the boundary condition again becomes the traditional continuous and anti-periodic boundary condition for $\chi^{S}$ :

$$
\begin{aligned}
& \chi^{A}\left(0^{+}\right)=-\chi^{A}\left(2 \beta^{-}\right), \quad \chi^{A}\left(2 \beta^{+}\right)=-\chi^{A}\left(4 \beta^{-}\right), \\
& \chi^{B}\left(0^{+}\right)=-\chi^{B}\left(4 \beta^{-}\right), \quad \chi^{B}\left(\beta^{-}\right)=\chi^{B}\left(3 \beta^{+}\right), \quad \chi^{B}\left(\beta^{+}\right)=-\chi^{B}\left(3 \beta^{-}\right) .
\end{aligned}
$$

Similar to the previous subsection, we consider the Green's function for $\chi^{S}$ :

$$
G^{A / B}\left(s, s^{\prime}\right)=\left\langle\mathcal{T}_{\mathcal{C}} \chi^{A / B}(s) \chi^{A / B}\left(s^{\prime}\right)\right\rangle
$$

The Schwinger-Dyson equation then reads

$$
\begin{aligned}
& G^{A}\left(s, s^{\prime}\right)=\left(\partial_{\tau}-\Sigma^{A}\right)_{A}^{-1}\left(s, s^{\prime}\right), \quad G^{B}\left(s, s^{\prime}\right)=\left(\partial_{\tau}-\Sigma^{B}\right)_{B}^{-1}\left(s, s^{\prime}\right), \\
& \Sigma^{A}\left(s, s^{\prime}\right)=\Sigma^{B}\left(s, s^{\prime}\right)=J^{2}\left(\lambda G^{A}\left(s, s^{\prime}\right)+(1-\lambda) G^{B}\left(s, s^{\prime}\right)\right)^{q-1} P^{(2)}\left(s, s^{\prime}\right) .
\end{aligned}
$$


Here the $A / B$ labels different boundary conditions for $A / B$ subsystem. This self-energy can be directly understood by the melon diagram (Here $q=4$ for example):

$$
\Sigma^{A}\left(s, s^{\prime}\right)=\Sigma^{B}\left(s, s^{\prime}\right)=\frac{\overbrace{A / B}^{\prime-\bar{A} / B^{\prime}}}{A / B} \sum_{s^{\prime}}^{\prime} .
$$

with $\lambda$ or $1-\lambda$ being the probability of having a mode in subsystem $A$ or $B$. We have $P^{(2)}\left(s, s^{\prime}\right)=1$ if both $\chi^{S}(s)$ and $\chi^{S}\left(s^{\prime}\right)$ represents the same field $\left(\chi_{1}^{S}\right.$ or $\left.\chi_{2}^{S}\right)$ and otherwise zero. Note that the main difference between this expression of self-energy and that for computing the subsystem Rényi entropy of thermal ensembles [37] is the presence of $P^{(2)}$.

These set of equations for $G^{A / B}$ and $\Sigma^{A / B}$ can also be directly derived by writing out the $G-\Sigma$ action and taking the saddle point approximation. Consequently, after solving (3.19), we have $\operatorname{tr}_{A} \tilde{\rho}_{A}^{2}=e^{-I^{(2)}}$ with:

$$
\begin{aligned}
\frac{I^{(2)}}{N}= & \frac{\lambda}{4} \log \operatorname{det} G^{A}\left(s, s^{\prime}\right)+\frac{\lambda(q-1)}{4 q} \int d s d s^{\prime} G^{A}\left(s, s^{\prime}\right) \Sigma^{A}\left(s, s^{\prime}\right) \\
& +\frac{1-\lambda}{4} \log \operatorname{det} G^{B}\left(s, s^{\prime}\right)+\frac{(1-\lambda)(q-1)}{4 q} \int d s d s^{\prime} G^{B}\left(s, s^{\prime}\right) \Sigma^{B}\left(s, s^{\prime}\right) .
\end{aligned}
$$

We have $I^{(2)}(\lambda=0)=I^{(2)}(\lambda=1)=2 I_{\mathrm{KM}}$. The second Rényi entanglement entropy is then given by

$$
\mathcal{S}_{A}^{(2)}=-\log \left(\operatorname{tr}_{A} \rho_{A}^{2}\right)=-\log \left(\frac{\operatorname{tr}_{A} \tilde{\rho}_{A}^{2}}{Z_{\mathrm{KM}}^{2}}\right)=I^{(2)}-2 I_{\mathrm{KM}} .
$$

The generalization of above discussions to $n$-th Rényi entropy is straightforward.

\section{Numerical results}

Because of the lack of translational invariance, the analytical study of (3.19) is difficult. In this section, we present numerical results for the entanglement entropy with different $q$ and $T / J$.

We perform the numerical iteration of (3.19) similar to that in $[37,40]$. We discretize the time $s$ into $L$ points, with $d s=4 \beta / L$. For $\beta=50$, we typically take $L \sim 400 \sim 600$. The equation (3.19) then becomes a matrix equation:

$$
\begin{aligned}
& \left(G^{A}\right)_{i j}=\left(\left(G_{0}^{A}\right)^{-1}-\Sigma^{A}\right)_{i j}^{-1}, \quad\left(G^{B}\right)_{i j}=\left(\left(G_{0}^{B}\right)^{-1}-\Sigma^{B}\right)_{i j}^{-1}, \\
& \left(\Sigma^{A}\right)_{i j}=\left(\Sigma^{B}\right)_{i j}=J^{2} d s^{2}\left(\lambda\left(G^{A}\right)_{i j}+(1-\lambda)\left(G^{B}\right)_{i j}\right)^{q-1} P_{i j}^{(2)} .
\end{aligned}
$$

Here $G_{0}^{A}$ and $G_{0}^{B}$ are the Green's functions without interaction $J$ on the contour (3.17). Their elements are either $\pm 1 / 2$ or 0 depending on the time ordering and the connectivity of contours. Explicitly:

$$
\begin{array}{rlrl}
\left(G_{0}^{A}\right)_{i j}=\frac{1}{2} \operatorname{sign}(i-j) & & \text { for }\{i, j\} \subset[1, L / 2] \text { or }[L / 2+1, L], \\
\left(G_{0}^{B}\right)_{i j}=\frac{1}{2} \operatorname{sign}(i-j) & \text { for }\{i, j\} \subset[1, L / 4] \cup[3 L / 4+1, L] \text { or }[L / 4+1,3 L / 4] .
\end{array}
$$



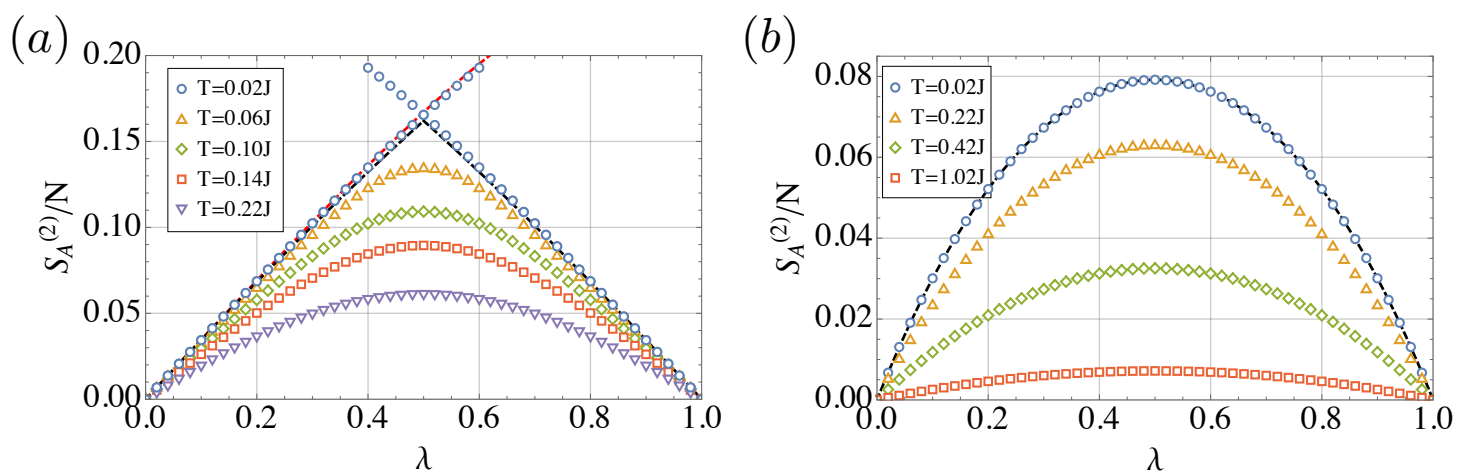

Figure 1. (a) The entanglement entropy $\mathcal{S}_{A}^{(2)} / N$ of KM states at different temperature $T / J$ with $q=4$. The black dashed line is the analytical approximation for $(2.4): \mathcal{S}_{A}^{(2)}(\lambda) / N=x(\log (2) / 2-$ $\left.\arcsin \left(x^{3 / 2}\right) / 16\right)$ with $x=\min \{\lambda, 1-\lambda\}$. The red dashed line is the subsystem entropy for a thermal ensemble with $\beta J=50$ [37]. (b) The entanglement entropy $\mathcal{S}_{A}^{(2)} / N$ of KM states at different temperature $T / J$ with $q=2$. The black dashed line is the analytical formula for the $\mathrm{SYK}_{2}$ ground state [37].

The on-shell action is then

$$
\begin{aligned}
\frac{I^{(2)}}{N}= & \frac{\lambda}{4} \log \operatorname{det}\left[G^{A}\left(G_{0}^{A}\right)^{-1}\right]+\frac{\lambda(q-1)}{4 q} \operatorname{tr}\left[G^{A}\left(\Sigma^{A}\right)^{T}\right]-\frac{1}{2} \log 2 \\
& +\frac{1-\lambda}{4} \log \operatorname{det}\left[G^{B}\left(G_{0}^{B}\right)^{-1}\right]+\frac{(1-\lambda)(q-1)}{4 q} \operatorname{tr}\left[G^{B}\left(\Sigma^{B}\right)^{T}\right] .
\end{aligned}
$$

Here we have used $\log \operatorname{det}\left(G_{0}^{A}\right)^{-1}=\log \operatorname{det}\left(G_{0}^{B}\right)^{-1}=2 \log 2$ to enable the convergence. The extrapolation towards $1 / L \rightarrow 0$ is performed finally.

Now we present numerical results for the entanglement entropy of KM pure states. We first focus on the $\mathrm{SYK}_{q}$ model with $q=4$ or $q=2$ as an example of strongly interacting systems or non-interacting systems.

The result of $\mathcal{S}_{A}^{(2)}$ for different $\beta J$ with $q=4$ is shown in figure 1 (a). We have also plotted the analytical approximation [34] as the black dashed line and the subsystem entropy for a thermal ensemble with $\beta J=50$ [37] as the red dashed line. For small $\beta J$, the entanglement builds up quickly as $\beta J$ increases, and $\mathcal{S}_{A}^{(2)} / N$ is an analytical function of $\lambda$. On the other side, for large $\beta J \gtrsim 30$ near $\lambda \sim 1 / 2$, there exist two different saddle point solutions, and the coexistence region becomes larger as $\beta J$ increases. The true curve for $\mathcal{S}_{A}^{(2)}$ is determined by the comparing actions of different saddles, leading a first-order transition. As we will see in the next section, these two saddle points smoothly connected to the subsystem entropy $\mathcal{S}_{A, \text { th }}^{(2)}$ or $\mathcal{S}_{B, \text { th }}^{(2)}$ of a thermal ensemble at corresponding temperature. For $T / J=0.02$, the numerical result of the KM state gives $\mathcal{S}_{A}^{(2)}(1 / 2)=0.331 M$, which is larger than the analytical approximation $\mathcal{S}_{A}^{(2)}(1 / 2)=0.324 M$, but also a little smaller than the thermal ensemble result $\mathcal{S}_{A}^{(2)}(1 / 2)=0.334 M$. We attribute this to the fact that KM states are non-thermal. This is to be compared with the quadratic Hamiltonian case with $q=2$ shown in figure $1(\mathrm{~b})$, where $\mathcal{S}_{A}^{(2)}(\lambda)$ is analytical even for very low temperature.

The existence of the transition gives rise to the singularity of the Page curve at $\lambda=$ $1 / 2$ [45], as expected for general chaotic systems. Consequently, the existence of transition 

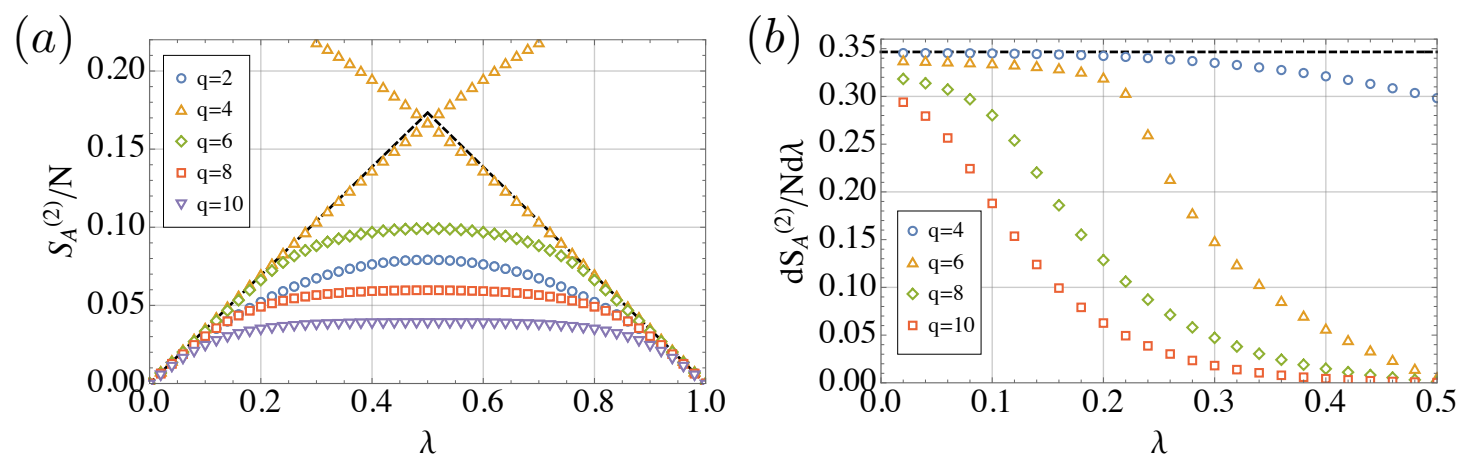

Figure 2. (a) The entanglement entropy $\mathcal{S}_{A}^{(2)} / N$ of KM states at different $q$ with $\beta \mathcal{J}=50$. The black dashed line is the maximal entropy: $\mathcal{S}_{A}^{(2)}(\lambda) / N=x \log (2) / 2$ with $x=\min \{\lambda, 1-\lambda\}$. (b) The derivative of the entanglement entropy $d \mathcal{S}_{A}^{(2)} / N d \lambda$ of KM states at different $q$ with $\beta \mathcal{J}=50$. The black dashed line represent the maximal entropy $\log (2) / 2$.

in highly entangled pure states to be a general feature for interacting systems with saddlepoint description, including different generalizations of the SYK model.

We then consider $q$ dependence for $\mathcal{S}_{A}^{(2)}$. Here we fix $\beta \mathcal{J}=50$. The numerical results are shown in figure 2 (a). The $q=4$ and $q=2$ case has been discussed above. If we further increase $q \geq 6$, we find the entanglement entropy decreases rapidly as $q$ increases. This can be understood that in the large $q$ limit, the system is weakly interacting. As a result, for fixed $\beta \mathcal{J}$ the system becomes less entangled as $q$ increases. It is also interesting to notice that for larger $q, \mathcal{S}_{A}^{(2)}$ becomes much more flat near $\lambda \sim 1 / 2$, which can also be seen from figure 2 (b). There is an analogy phenomenon for the entanglement entropy under the random Hamiltonian evolution [49]. It is also reasonable that for larger $q$, the Hamiltonian becomes denser and resembles a random Hamiltonian.

\section{Quench dynamics for pure-state entanglement entropy}

We now turn to the study of real-time entanglement dynamics by considering the evolution of $|\mathrm{KM}\rangle$. The normalized state after evolving time $t$ is

$$
|\psi(t)\rangle=\frac{1}{\sqrt{Z_{\mathrm{KM}}}} e^{-i H t}|\mathrm{KM}\rangle .
$$

We first consider the two-point function of $\chi_{i}$ on real-time. There are studies on quench dynamics for two-point functions for different SYK-like models in the large- $N$ limit [50$54]$ by solving the Kadanoff-Baym equation [55]. ${ }^{5}$ However, in our case the two point function can be directly obtained by an analytical continuation of (2.6). Consequently, the diagonal components $G_{i i}$ are again thermal at any time while the off-diagonal components $G_{2 j-1,2 j}(t) \sim G_{\mathrm{th}}(\beta / 2)^{2} e^{-2 v t}$ in the long-time limit. Here $v$ is the decay rate of the real-time two-point function on thermal ensemble. In the low-temperature limit, we have $v \sim 1 / \beta$ and $G_{\mathrm{th}}(\beta / 2) \rightarrow 0$. In the high-temperature limit, we instead have $v \sim J$, which leads to a thermalization time $t_{\text {th }} \sim 1 / J$.

\footnotetext{
${ }^{5}$ In these works, the initial state is a thermal ensemble. Here we instead of focus on pure states.
} 
We would like to study this quenching process from the entanglement perspective. To compute the evolution of the entanglement entropy, we again apply the path integral representation using the contour in (3.17). The main difference is that now the solid lines can be either (forward/backward) real or imaginary-time evolution:

$$
\operatorname{tr}_{A} \rho_{A}(t)^{2}=\operatorname{tr}_{A}\left(\operatorname{tr}_{B}|\psi(t)\rangle\langle\psi(t)|\right)^{2}=\frac{1}{Z_{\mathrm{KM}}^{2}} \times
$$

Here we draw an arrow for the direction of real-time evolutions. When the parametrization is along the same direction as the arrow, the real-time evolution is effectively forward. Otherwise, the evolution is backward. Since the evolution is governed by $e^{-\tau H}$ for imaginary-time evolution and $e^{\mp i H t}$ for forward/backward real-time evolution, we need to add additional factor of $\pm i$. This leads to the modification of (3.19):

$$
\begin{aligned}
& G^{A}\left(s, s^{\prime}\right)=\left(\partial_{\tau}-\Sigma^{A}\right)_{A}^{-1}\left(s, s^{\prime}\right), \quad G^{B}\left(s, s^{\prime}\right)=\left(\partial_{\tau}-\Sigma^{B}\right)_{B}^{-1}\left(s, s^{\prime}\right), \\
& \Sigma^{A}\left(s, s^{\prime}\right)=\Sigma^{B}\left(s, s^{\prime}\right)=J^{2}\left(\lambda G^{A}\left(s, s^{\prime}\right)+(1-\lambda) G^{B}\left(s, s^{\prime}\right)\right)^{q-1} \tilde{P}^{(2)}\left(s, s^{\prime}\right) .
\end{aligned}
$$

where now we have $s \in[0,4(\beta+2 t))$, including both the real-time and the imaginary-time evolution. Here $\tilde{P}^{(2)}\left(s, s^{\prime}\right)=P^{(2)}\left(s, s^{\prime}\right) f(s) f\left(s^{\prime}\right)$ with $f(s)=1, i$ or $-i$ for $s$ being a parameter of an imaginary-/forward real- or backward real-time evolution. After solving (5.3), the on-shell action can still be computed as (3.21).

We first consider the case with $q \geq 4$. Since the system satisfy the ETH, we expect when $\lambda<1 / 2$ the entanglement entropy approaches the subsystem entropy of a thermal ensemble in the long time limit. This can be understood by expanding

$$
|\psi(t)\rangle=\sum_{m} c_{m} e^{-i E_{m} t}\left|E_{m}\right\rangle .
$$

Here $\left|E_{m}\right\rangle$ are eigenstates of the Hamiltonian with eigenenergy $E_{m}$. The entanglement entropy for a subsystem $A$ can be written as the expectation of the swap operator on two replicas of the original system:

$$
e^{-\mathcal{S}_{A}^{(2)}(t)}=\operatorname{tr}_{A}\left(\operatorname{tr}_{B}|\psi(t)\rangle\langle\psi(t)|\right)^{2}=\operatorname{tr}\left[\hat{S}^{A}|\psi(t)\rangle\langle\psi(t)|\otimes| \psi(t)\rangle\langle\psi(t)|\right]
$$

Here $\hat{S}^{A}$ is the swap operator of subsystem $A$ on doubled Hilbert space. Consequently, in the long time limit, we have

$$
\begin{aligned}
e^{-\mathcal{S}_{A}^{(2)}} & \approx \sum_{m, n}\left|c_{m} c_{n}\right|^{2} \operatorname{tr}\left[\hat{S}^{A}\left(\left|E_{m}\right\rangle\left\langle E_{m}|\otimes| E_{n}\right\rangle\left\langle E_{n}|+| E_{n}\right\rangle\left\langle E_{m}|\otimes| E_{m}\right\rangle\left\langle E_{n}\right|\right)\right] \\
& =\sum_{m, n}\left|c_{m} c_{n}\right|^{2} \operatorname{tr}\left[\left(\hat{S}^{A}+\hat{S}^{B}\right)\left|E_{m}\right\rangle\left\langle E_{m}|\otimes| E_{n}\right\rangle\left\langle E_{n}\right|\right] \\
& =\operatorname{tr}\left[\left(\hat{S}^{A}+\hat{S}^{B}\right) \rho_{\mathrm{th}} \otimes \rho_{\mathrm{th}}\right]=e^{-\mathcal{S}_{A, t h}^{(2)}+e^{-\mathcal{S}_{B, t h}^{(2)}}}
\end{aligned}
$$


(a)

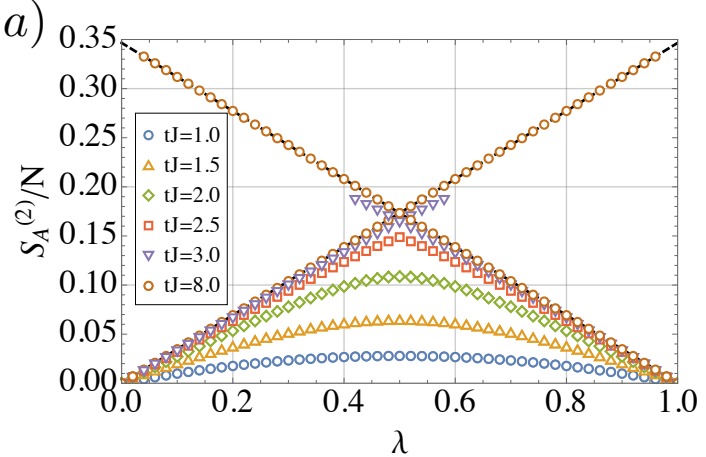

(b)

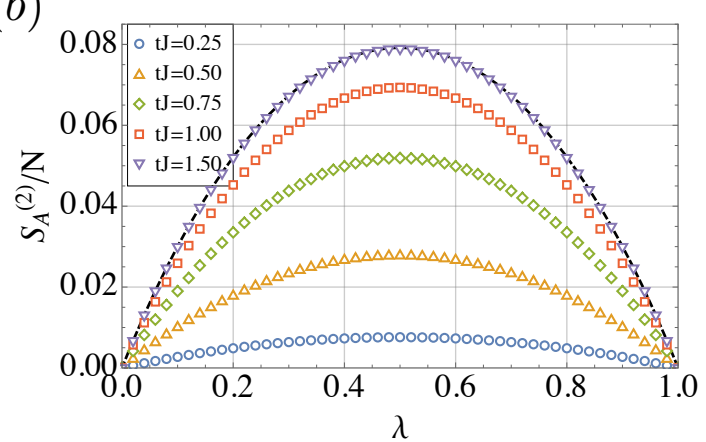

Figure 3. (a) The dynamics of the Rényi entanglement entropy $\mathcal{S}_{A}^{(2)}(t)$ as a function of subsystem size $\lambda$ with $q=4$ and $\beta=0$. The black dashed reference line is $\lambda \log (2) / 2$ and $(1-\lambda) \log (2) / 2$. (b) The dynamics of the Rényi entanglement entropy $\mathcal{S}_{A}^{(2)}(t)$ as a function of subsystem size $\lambda$ with $q=2$ and $\beta=0$. The black dashed line is the analytical formula for the $\mathrm{SYK}_{2}$ ground state [37].

We have used the fact that the diagonal ensemble $\sum_{n}\left|c_{n}\right|^{2}\left|E_{n}\right\rangle\left\langle E_{n}\right|$ is approximately a thermal ensemble $\rho_{\text {th }}$ (of the full system) with inverse temperature $\beta$ for systems satisfying the ETH. Previous study shows $\mathcal{S}_{A, t h}^{(2)}<\mathcal{S}_{B, t h}^{(2)}$ for $\lambda<1 / 2$ while $\mathcal{S}_{A, t h}^{(2)}>\mathcal{S}_{B, t h}^{(2)}$ for $\lambda>$ $1 / 2$ [37]. Note that this is a non-trival statement since the effective temperature depends on $\lambda$ for non-local Hamiltonians (2.4). Consequently, in the large- $N$ limit, we have $\mathcal{S}_{A}^{(2)}=\mathcal{S}_{A, t h}^{(2)}$ for $\lambda<1 / 2$ and $\mathcal{S}_{A}^{(2)}=\mathcal{S}_{B, t h}^{(2)}$ for $\lambda>1 / 2$.

The numerical result for $\mathcal{S}_{A}^{(2)}(t)$ with $q=4$ is shown in figure 3 (a). Here we have set $\beta=0$. Similar to tunning the imaginary-time $\beta$, we find a first-order transition in the long real-time limit. By comparing with $\lambda \log (2) / 2$ and $(1-\lambda) \log (2) / 2$, two different saddles in the long-time limit can be identified with the contribution from $e^{-\mathcal{S}_{A, t h}^{(2)}}$ or $e^{-\mathcal{S}_{B, t h}^{(2)}}$ in (5.5). We have also checked the match for finite $\beta$.

We could also understand the two saddle points directly from the path-integral representation (5.2). For convenience, here we put back the possible $\{s\}$ dependence of KM states. we write $|\{s\}\rangle=\left|\left\{s_{A}\right\}\right\rangle_{A} \otimes\left|\left\{s_{B}\right\}\right\rangle_{B}$. In the long-time limit, the saddle point solutions can be understood as follows: We first consider turn off the interaction between subsystem $A$ and $B$. Then the Green's functions $G^{A}$ and $G^{B}$ become block diagonal according to $G_{0}^{A} / G_{0}^{B}$. When we turn on the interaction between subsystem $A$ and $B$, one saddle is given by: We keep $G^{B}$ almost replica diagonal. Two half contours of $G^{A}$ that interacts with the same $B$ subsystem become effectively connected $[20,40,56]$, while two $\chi_{i}^{A}$ interact with different $B$ subsystems becomes less correlated far away from the boundary of the contour. Graphically, this means

Saddle point corresponds to $\mathcal{S}_{A, t h}^{(2)}$ :

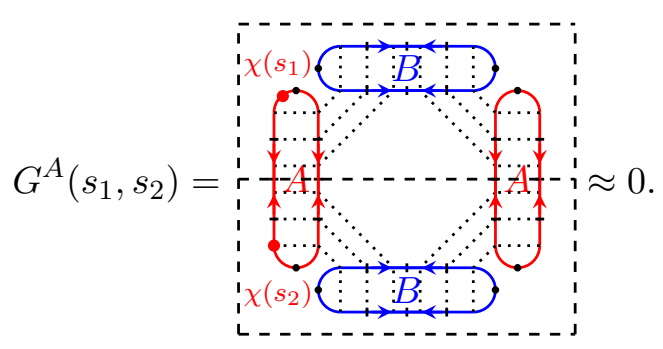


Here the contours within each box are effectively connected. The red dots represent the insertion of field $\chi^{A}$. An important observation is that since the correlation is small between two boxes near the black dots where the boundary condition is imposed, we could make the approximation:
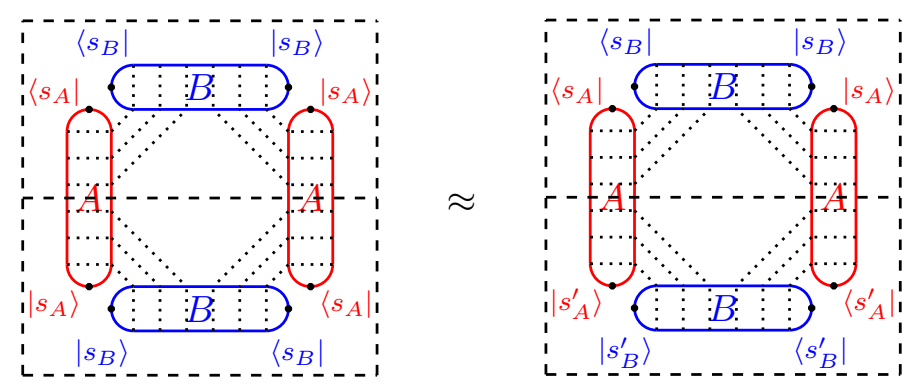

Here we drop the arrow for simplicity. Physically, this may be understood as follows: if we evolve for a long time, the reduced density matrix of subsystem $A$ always becomes thermal, and we can not distinguish different initial states $|\{s\}\rangle$ or $\left|\left\{s^{\prime}\right\}\right\rangle$. If we sum over all possible boundary states, the contour becomes:

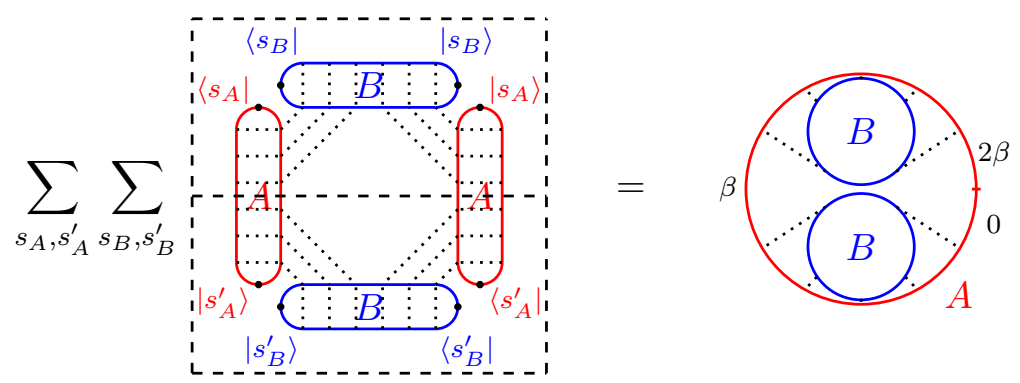

Here we have used the relation

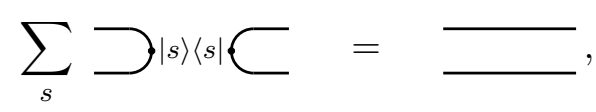

and we have merged the contour for $\chi_{1}^{S}$ and $\chi_{2}^{S}$ for both subsystem $S=A / B$. This is exactly the contour for computing the subsystem Rényi entropy $\mathcal{S}_{A, t h}^{(2)}$ of a thermal ensemble [37]. Similarly, if we exchange the role of $A$ and $B$ subsystem, we get another saddle point corresponds to $\mathcal{S}_{B, t h}^{(2)}$.

Note that this existence of the first-order transition can be viewed as an analogy of the information paradox in more complicated set-ups [15, 15-20, 40]: if we consider increasing $\lambda$ and time $t$ from $\lambda=0$ and $t=0$, we could follow the saddle of $e^{-\mathcal{S}_{A, t h}^{(2)}}$ without facing any singularity till $\lambda=1$, which leads to an information paradox. The solution to the information paradox is also similar: at some time $t$, a new saddle-point appears which preserves the unitary. For setups with clear bulk description, this new saddle corresponds to a solution with islands $[15,15-20]$.

For $q=2$, the system shows qualitatively different behaviors. Since the state $|\{1\}\rangle$ is annihilated by $c_{j},|\psi(t)\rangle$ is then annihilated by $c_{j}(\beta / 2+i t)$. For long time $t$, we expect this to be a random superposition $c_{j}(i \infty)=\sum_{i} O_{j i} \chi_{i}$ with some parameter $O_{j i}$. An remarkable 
(a)

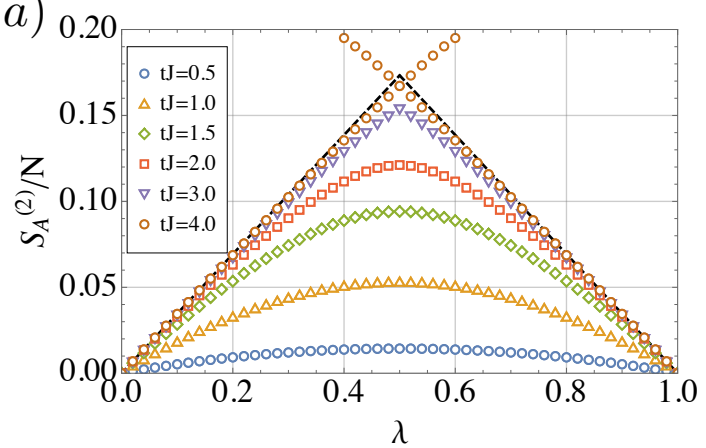

(b)

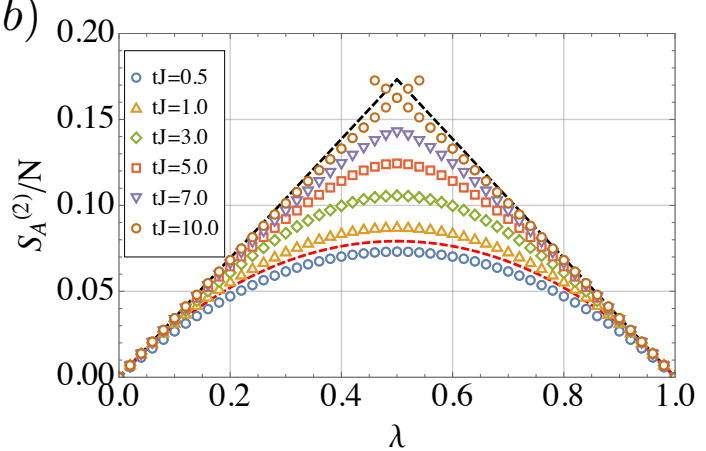

Figure 4. (a) The dynamics of the Rényi entanglement entropy $\mathcal{S}_{A}^{(2)}(t)$ as a function of subsystem size $\lambda$ with $V / J=1 / 2$ and $\beta=0$. The black dashed line is the maximal entropy: $\mathcal{S}_{A}^{(2)}(\lambda) / N=$ $x \log (2) / 2$ with $x=\min \{\lambda, 1-\lambda\}$. (b) The dynamics of the Rényi entanglement entropy $\mathcal{S}_{A}^{(2)}(t)$ as a function of subsystem size $\lambda$ with $V / J=2$ and $\beta=0$. The black dashed line is the maximal entropy $\mathcal{S}_{A}^{(2)}(\lambda) / N=x \log (2) / 2$ with $x=\min \{\lambda, 1-\lambda\}$. The black dashed line is the analytical formula for the $\mathrm{SYK}_{2}$ ground state [37].

observation is that this is just the ground state of $\mathrm{SYK}_{2}$ model, and the entanglement entropy $\mathcal{S}_{A}^{(2)}$ is again given in [37]. As shown in figure $3(\mathrm{~b})$, the arguments works even for $\beta=0$, which means the system would not thermalize to an infinite temperature ensemble $\rho=2^{-\frac{N}{2}} I$, as expected for non-interacting systems.

We further consider adding $\mathrm{SYK}_{4}$ random interaction term to the $\mathrm{SYK}_{2}$ random hopping model [57-59]. The Hamiltonian reads

$$
H_{V}=\frac{1}{4 !} \sum_{i j k l} J_{i j k l}^{(4)} \chi_{i} \chi_{j} \chi_{k} \chi_{l}+\frac{1}{2} \sum_{i j} i V_{i j}^{(2)} \chi_{i} \chi_{j} .
$$

Here to avoid possible confusion, we have changed the notion of the random hopping parameters to be $V_{i j}^{(2)}$ with variance $\overline{\left(V_{i j}^{(2)}\right)^{2}}=V^{2} / N$. Near the $\mathrm{SYK}_{4}$ fixed point, the random hopping term is relevant. As a result, the system is always a non-Fermi liquid for at low temperature $T \ll V^{2} / J$. At finite temperature, there is a crossover between the $\mathrm{SYK}_{2}$ and the $\mathrm{SYK}_{4}$ fixed points [58].

We could similarly define the KM pure states for this model and study the entanglement dynamics with minor modification of (5.3):

$$
\Sigma^{A}=\Sigma^{B}=\left[J^{2}\left(\lambda G^{A}+(1-\lambda) G^{B}\right)^{3}+V^{2}\left(\lambda G^{A}+(1-\lambda) G^{B}\right)\right] \tilde{P}^{(2)} .
$$

The evolution of $\mathcal{S}_{A}^{(2)}$ for different $V / J$ is shown in figure 4 . We find that the entanglement show different behaviors for $V \lesssim J$ and $V \gtrsim J$. For $V \lesssim J$ the behavior is basically the same as the $\mathrm{SYK}_{4}$ case in figure 3 (a), as shown in figure 4 (a). The reason is that the random hopping term plays an role only in the long time limit $t \sim J / V^{2}$, when the entanglement has already been built up.

This is to be compared with the $V \gtrsim J$ case shown in figure 4 (b). In this case, the system builds up the entanglement in two steps. The short time behavior is governed by the random hopping term, leading to a $\mathcal{S}_{A}^{(2)}$ close to the $\mathrm{SYK}_{2}$ ground state. Then the 
(a)

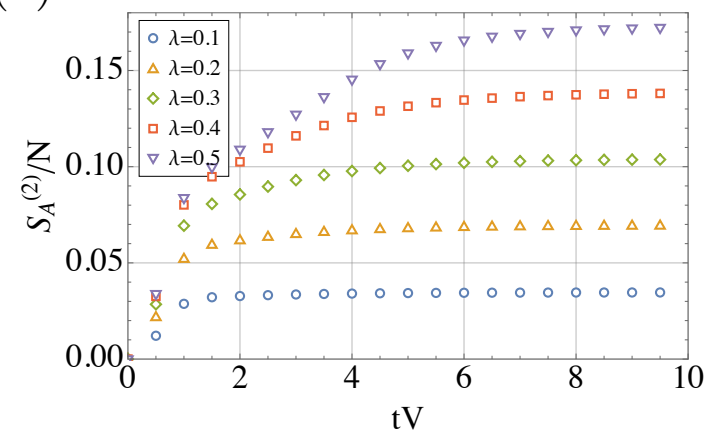

(b)

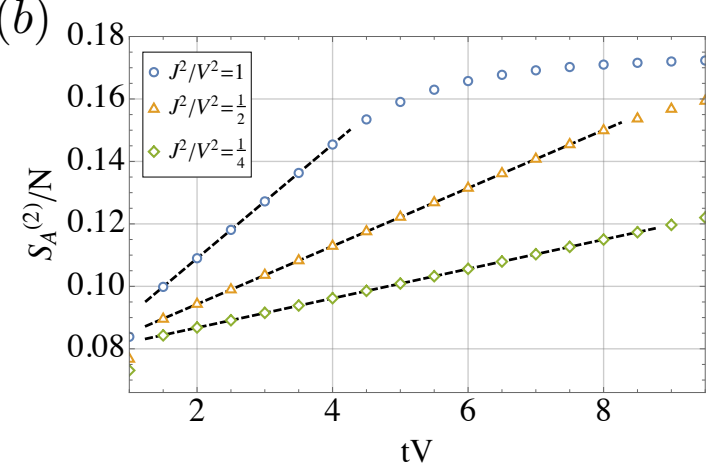

Figure 5. (a) The Rényi entanglement entropy $\mathcal{S}_{A}^{(2)}(t)$ as a function of time $t V$ for different subsystem size $\lambda$. Here we take $\beta=0$ and $V=J$. (b) The Rényi entanglement entropy $\mathcal{S}_{A}^{(2)}(t)$ as a function of time $t V$ for different subsystem size $V / J$. Here we fix $\beta=0$ and $\lambda=1 / 2$. The black dashed lines are linear fits for the linear growth region.

entanglement continues to increase linearly but with much slower speed until the system thermalizes. This can also been seen from a plot for $\mathcal{S}_{A}^{(2)}(t)$ with different time $t$. As shown in figure 5 (a), the entanglement entropy with $\lambda=1 / 2$ and $V=J$ increases rapidly for $t V<1$, while it increases linearly with a smaller slope for $t V>1$ until its saturation. The slope of the linear growth is proportional to $J^{2} / V$, which can be seen from figure 5 (b). This can be understood as a perturbative calculation near the $J=0$ solution, similar to the short-time behavior in $[39,40]$.

\section{Conclusion}

In this work, we study the entanglement Rényi entropy of Kourkoulou-Maldacena pure states of the SYK model, including its generalizations. We use the path-integral approach which gives the exact entanglement entropy in the large- $N$ limit.

At low energy density, we find a first-order transition for the entanglement entropy for the $\mathrm{SYK}_{4}$ model when tuning the subsystem size $\lambda$. This is different compared to the $q=2$ case where the entropy is a smooth function. Similar behaviors exist if we consider long real-time evolution. The first-order transition is from the existence of two different saddle points, which corresponds to the thermal Rényi entropy of reduced density matrices for different subsystems. We further consider adding small $\mathrm{SYK}_{4}$ random interaction to the $\mathrm{SYK}_{2}$ case, leading to the slow linear growth of entanglement entropy in the intermediate time regime.

\section{Acknowledgments}

We thank Xiao Chen, Yingfei Gu, Chunxiao Liu for discussion. PZ acknowledges support from the Walter Burke Institute for Theoretical Physics at Caltech. 
Open Access. This article is distributed under the terms of the Creative Commons Attribution License (CC-BY 4.0), which permits any use, distribution and reproduction in any medium, provided the original author(s) and source are credited.

\section{References}

[1] J.M. Deutsch, Quantum statistical mechanics in a closed system, Phys. Rev. A 43 (1991) 2046.

[2] M. Srednicki, Chaos and quantum thermalization, Phys. Rev. E 50 (1994) 888.

[3] P. Calabrese and J. Cardy, Entanglement entropy and quantum field theory, J. Stat. Mech. 06 (2004) P06002 [hep-th/0405152].

[4] J.R. Garrison and T. Grover, Does a single eigenstate encode the full Hamiltonian?, Phys. Rev. X 8 (2018) 021026 [arXiv: 1503.00729] [INSPIRE].

[5] R. Islam et al., Measuring entanglement entropy in a quantum many-body system, Nature $\mathbf{5 2 8}(2015) 77$.

[6] J. Li et al., Measuring out-of-time-order correlators on a nuclear magnetic resonance quantum simulator, Phys. Rev. X 7 (2017) 031011 [arXiv: 1609.01246] [INSPIRE].

[7] B. Bauer and C. Nayak, Area laws in a many-body localized state and its implications for topological order, J. Stat. Mech. 09 (2013) P09005.

[8] A. Lukin et al., Probing entanglement in a many-body-ocalized system, Science 364 (2019) 256.

[9] P. Hosur, X.L. Qi, D.A. Roberts and B. Yoshida, Chaos in quantum channels, JHEP 02 (2016) 004 [arXiv: 1511.04021].

[10] R. Fan et al., Out-of-time-order correlation for many-body localization, Sci. Bull. 62 (2017) 707 [arXiv: 1608.01914].

[11] N. Lashkari et al., Towards the fast scrambling conjecture, JHEP 04 (2013) 022 [arXiv:1111.6580].

[12] A. Kitaev, Hidden correlations in the Hawking radiation and thermal noise, talk given at the 2015 Breakthrough Prize Fundamental Physics Symposium, November 10, San Francisco U.S.A. (2015).

[13] W.W. Ho and D.A. Abanin, Entanglement dynamics in quantum many-body systems, Phys. Rev. B 95 (2017) 094302 [arXiv: 1508. 03784] [INSPIRE].

[14] M. Mezei and D. Stanford, On entanglement spreading in chaotic systems, JHEP 05 (2017) 065 .

[15] G. Penington, Entanglement wedge reconstruction and the information paradox, arXiv: 1905.08255 [INSPIRE].

[16] A. Almheiri, N. Engelhardt, D. Marolf and H. Maxfield, The entropy of bulk quantum fields and the entanglement wedge of an evaporating black hole, JHEP 12 (2019) 063 [arXiv: 1905.08762] [INSPIRE].

[17] A. Almheiri, R. Mahajan, J. Maldacena and Y. Zhao, The Page curve of Hawking radiation from semiclassical geometry, JHEP 03 (2020) 149 [arXiv: 1908.10996] [INSPIRE]. 
[18] A. Almheiri, R. Mahajan and J. Maldacena, Islands outside the horizon, arXiv:1910.11077 [INSPIRE].

[19] A. Almheiri et al., Replica wormholes and the entropy of Hawking radiation, JHEP 05 (2020) 013 [arXiv : 1911.12333] [INSPIRE].

[20] G. Penington, S.H. Shenker, D. Stanford and Z. Yang, Replica wormholes and the black hole interior, arXiv: 1911.11977 [INSPIRE].

[21] S. Ryu and T. Takayanagi, Holographic derivation of entanglement entropy from AdS/CFT, Phys. Rev. Lett. 96 (2006) 181602 [hep-th/0603001] [INSPIRE].

[22] S. Ryu and T. Takayanagi, Aspects of holographic entanglement entropy, JHEP 08 (2006) 045 [hep-th/0605073] [INSPIRE].

[23] A. Lewkowycz and J. Maldacena, Generalized gravitational entropy, JHEP 08 (2013) 090 [arXiv: 1304.4926] [INSPIRE].

[24] P. Hayden et al., Holographic duality from random tensor networks, JHEP 11 (2016) 009 [arXiv: 1601.01694].

[25] A. Nahum, J. Ruhman, S. Vijay and J. Haah, Quantum entanglement growth under random unitary dynamics, Phys. Rev. X 7 (2017) 031016 [arXiv: 1608.06950] [INSPIRE].

[26] C. von Keyserlingk, T. Rakovszky, F. Pollmann and S. Sondhi, Operator hydrodynamics, OTOCs and entanglement growth in systems without conservation laws, Phys. Rev. X 8 (2018) 021013 [arXiv: 1705.08910] [INSPIRE].

[27] S. Sachdev and J. Ye, Gapless spin fluid ground state in a random, quantum Heisenberg magnet, Phys. Rev. Lett. 70 (1993) 3339 [cond-mat/9212030] [INSPIRE].

[28] J. Maldacena and D. Stanford, Remarks on the Sachdev-Ye-Kitaev model, Phys. Rev. D 94 (2016) 106002 [arXiv: 1604.07818] [INSPIRE].

[29] A. Kitaev and S.J. Suh, The soft mode in the Sachdev-Ye-Kitaev model and its gravity dual, JHEP 05 (2018) 183 [arXiv: 1711.08467].

[30] J. Maldacena, D. Stanford and Z. Yang, Conformal symmetry and its breaking in two-dimensional nearly Anti-de Sitter space, Prog. Theor. Phys. (2016) 12C104 [arXiv: 1606.01857].

[31] N. Hunter-Jones, J. Liu and Y. Zhou, On thermalization in the SYK and supersymmetric syk models, JHEP 02 (2018) 142 [arXiv:1710.03012].

[32] M. Haque and P. McClarty, Eigenstate thermalization scaling in Majorana clusters: From chaotic to integrable Sachdev-Ye-Kitaev models, Phys. Rev. B 100 (2019) 115122 [arXiv: 1711.02360] [INSPIRE].

[33] J. Sonner and M. Vielma, Eigenstate thermalization in the Sachdev-Ye-Kitaev model, JHEP 11 (2017) 149 [arXiv:1707.08013].

[34] Y. Huang and Y. Gu, Eigenstate entanglement in the Sachdev-Ye-Kitaev model, Phys. Rev. D 100 (2017) 041901 [arXiv:1709.09160] [InSPIRE].

[35] A.M. García-García and J.J.M. Verbaarschot, Analytical spectral density of the Sachdev-Ye-Kitaev model at finite N, Phys. Rev. D 96 (2017) 066012 [arXiv:1701.06593] [INSPIRE].

[36] C. Liu, X. Chen and L. Balents, Quantum entanglement of the Sachdev-Ye-Kitaev models, Phys. Rev. B 97 (2018) 245126 [arXiv:1709.06259] [INSPIRE]. 
[37] P. Zhang, C. Liu and X. Chen, Subsystem Rényi entropy of thermal ensembles for SYK-like models, arXiv:2003.09766 [INSPIRE].

[38] A. Haldar, S. Bera and S. Banerjee, Renyi entanglement entropy of Fermi liquids and non-Fermi liquids: Sachdev-Ye-Kitaev model and dynamical mean field theories, arXiv:2004.04751 [INSPIRE].

[39] Y. Gu, A. Lucas and X.-L. Qi, Spread of entanglement in a Sachdev-Ye-Kitaev chain, JHEP 09 (2017) 120 [arXiv: 1708.00871].

[40] Y. Chen, X.-L. Qi and P. Zhang, Replica wormhole and information retrieval in the SYK model coupled to Majorana chains, arXiv:2003.13147 [INSPIRE].

[41] J. M. Magán, Black holes as random particles: entanglement dynamics in infinite range and matrix models, JHEP 08 (2016) 081 [arXiv: 1601.04663].

[42] J.M. Magan, Random free fermions: an analytical example of eigenstate thermalization, Phys. Rev. Lett. 116 (2016) 030401 [arXiv:1508.05339] [INSPIRE].

[43] J.M. Magan, De Finetti theorems and entanglement in large- $N$ theories and gravity, Phys. Rev. D 96 (2017) 086002 [arXiv: 1705. 03048] [INSPIRE].

[44] I. Kourkoulou and J. Maldacena, Pure states in the SYK model and nearly-AdS $S_{2}$ gravity, arXiv: 1707.02325 [INSPIRE].

[45] D.N. Page, Average entropy of a subsystem, Phys. Rev. Lett. 71 (1993) 1291 [gr-qc/9305007] [INSPIRE].

[46] W. Fu and S. Sachdev, Numerical study of fermion and boson models with infinite-range random interactions, Phys. Rev. B 94 (2016) 035135 [arXiv: 1603.05246] [INSPIRE].

[47] G. Gur-Ari, R. Mahajan and A. Vaezi, Does the SYK model have a spin glass phase?, JHEP 11 (2018) 070 [arXiv: 1806.10145].

[48] Y. Gu, A. Kitaev, S. Sachdev and G. Tarnopolsky, Notes on the complex Sachdev-Ye-Kitaev model, JHEP 02 (2020) 157 [arXiv: 1910.14099] [INSPIRE].

[49] Y.-Z. You and Y. Gu, Entanglement features of random hamiltonian dynamics, Phys. Rev. B 98 (2018) 014309 [arXiv: 1803.10425] [INSPIRE].

[50] A. Eberlein, V. Kasper, S. Sachdev and J. Steinberg, Quantum quench of the Sachdev-Ye-Kitaev model, Phys. Rev. B 96 (2017) 205123 [arXiv:1706.07803] [INSPIRE].

[51] A. Haldar, P. Haldar, S. Bera, I. Mandal and S. Banerjee, Quench, thermalization and residual entropy across a non-Fermi liquid to Fermi liquid transition, Phys. Rev. Res. 2 (2020) 013307 [arXiv: 1903.09652] [INSPIRE].

[52] C. Kuhlenkamp and M. Knap, Periodically driven Sachdev-Ye-Kitaev models, Phys. Rev. Lett. 124 (2020) 106401 [arXiv: 1906.06341] [INSPIRE].

[53] P. Zhang, Evaporation dynamics of the Sachdev-Ye-Kitaev model, Phys. Rev. B 100 (2019) 245104 [arXiv: 1909.10637] [INSPIRE].

[54] A. Almheiri, A. Milekhin and B. Swingle, Universal Constraints on Energy Flow and SYK Thermalization, arXiv:1912.04912 [INSPIRE].

[55] G. Stefanucci and R. Van Leeuwen, Nonequilibrium many-body theory of quantum systems: a modern introduction. Cambridge University Press, Cambridge U.K. (2013). 
[56] P. Saad, S.H. Shenker and D. Stanford, A semiclassical ramp in SYK and in gravity, arXiv: 1806.06840 [INSPIRE].

[57] S. Banerjee and E. Altman, Solvable model for a dynamical quantum phase transition from fast to slow scrambling, Phys. Rev. B 95 (2017) 134302 [arXiv:1610.04619] [InSPIRE].

[58] X. Chen, R. Fan, Y. Chen, H. Zhai and P. Zhang, Competition between Chaotic and Nonchaotic Phases in a Quadratically Coupled Sachdev-Ye-Kitaev Model, Phys. Rev. Lett. 119 (2017) 207603 [arXiv: 1705. 03406] [INSPIRE].

[59] X.-Y. Song, C.-M. Jian and L. Balents, Strongly correlated metal built from Sachdev-Ye-Kitaev models, Phys. Rev. Lett. 119 (2017) 216601 [arXiv:1705.00117] [INSPIRE]. 